\begin{tabular}{|c|c|}
\hline Title & Theoretical modeling of spin quantum cross structure devices with noncollinear ferromagnetic electrodes \\
\hline Author(s) & Kondo, Kenji \\
\hline Citation & $\begin{array}{l}\text { Journal of applied physics, } 107(9), 09 C 709-1-09 C 709-3 \\
\text { https://doi.org/10.1063/1.3357330 }\end{array}$ \\
\hline Issue Date & 2010-05-01 \\
\hline Doc URL & http:/hdl.handle.net/2115/56990 \\
\hline Rights & $\begin{array}{l}\text { Copyright } 2010 \text { A merican Institute of Physics. This article may be downloaded for personal use only. A ny other use } \\
\text { requires prior permission of the author and the A merican Institute of Physics. The following article appeared in Journal } \\
\text { of A pplied Physics and may be found at http://scitation.aip.org/content/aip } \$ \text { ournal } \$ \text { ap } / 107 / 9 / 10.1063 / 1.3357330 \text {. }\end{array}$ \\
\hline Type & article \\
\hline File Information & JAP_107_09C709_1.3357330.pdf \\
\hline
\end{tabular}

Instructions for use 


\title{
Theoretical modeling of spin quantum cross structure devices with noncollinear ferromagnetic electrodes
}

\author{
Kenji Kondo ${ }^{\text {a) }}$ \\ Laboratory of Quantum Electronics, Research Institute for Electronic Science, Hokkaido University, \\ Sapporo, Hokkaido 001-0020, Japan
}

(Presented 21 January 2010; received 26 October 2009; accepted 8 December 2009; published online 3 May 2010)

\begin{abstract}
Recently, we have proposed a spin quantum cross structure (SQCS) device toward the realization of novel switching devices. The SQCS device consists of two ferromagnetic metal thin films with their edges facing each other at an angle of $\theta$, and sandwiches a few molecules and atoms. In this paper, the calculation of electronic transport has been performed for SQCS devices with the Ni noncollinear magnetic films as both electrodes within the framework of the Anderson Hamiltonian, taking into consideration both polar angle $\theta$, and azimuthal angle $\phi$. We have obtained the general noncollinear spin transport formula, and the polar angle dependence of current-voltage characteristics of SQCS devices. The noncollinear spin transport is determined only by the angle $\theta$ defined by the inner product of two spins. Also, it is implied that SQCS devices can serve as multivalued memory devices by varying the angle $\theta$. (C) 2010 American Institute of Physics.

[doi:10.1063/1.3357330]
\end{abstract}

\section{INTRODUCTION}

We have proposed a spin quantum cross structure (SQCS) device as a candidate beyond complementary metaloxide semiconductor. ${ }^{1-4}$ The SQCS device consists of two ferromagnetic metal thin films with their edges facing each other at an angle of $\theta$ as shown in Figs. 1(a) and 1(b). The SQCS device has potential applications in both spin dependent switching devices and spin dependent high-density memory devices by sandwiching a few molecules and atoms. Also, the SQCS device has favorable configuration for investigating the spin dependent electronic transport between the noncollinear ferromagnetic electrodes because we can easily change the direction of magnetization in both electrodes by rotating the other electrode by an angle of $\theta$. This fact means that the calculation results can be easily verified by experiments. The ferromagnetic metal thin films such as $\mathrm{Ni}, \mathrm{Fe}$, and Co can be made by an evaporation method on organic substrates. Then, we can realize SQCS devices by using the edges of evaporated ferromagnetic metal thin films as both electrodes. In previous work, we studied the spin dependent electronic transport of the SQCS devices with the Ni collinear magnetic films as both electrodes, theoretically and experimentally. ${ }^{4}$ In this paper, we investigate the general spin dependent electronic transport of the SQCS devices with the $\mathrm{Ni}$ noncollinear magnetic films as both electrodes within the framework of the Anderson Hamiltonian, taking into consideration both polar angle $\theta$, and azimuthal angle $\phi$.

\section{THEORY AND RESULTS}

We study the current-voltage $(I-V)$ characteristics of SQCS devices with a molecule sandwiched between two Ni noncollinear magnetic electrodes. The molecule is assumed to have two energy levels. We include the possibility that the

${ }^{a)}$ Electronic mail: kkondo@es.hokudai.ac.jp. molecule flips the spin of an electron passing through the energy level. The model of SQCS devices with twodimensional (2D) electrodes is shown in Fig. 1(c). The energy diagram for the SQCS device model is also shown in Fig. 1(d). We analyze the transport characteristics using the Anderson Hamiltonian. The Anderson Hamiltonian is described as follows:

$$
H=H_{\text {Electrodes }}+H_{\text {mole }}+H_{\mathrm{t}},
$$

where

$$
\begin{aligned}
& H_{\text {Electrodes }}=\sum_{\alpha=L, R} \sum_{k, \sigma} \epsilon_{\alpha k \sigma} c_{\alpha k \sigma}^{\dagger} c_{\alpha k \sigma}, \\
& H_{\text {mole }}=\sum_{i, \sigma} \epsilon_{0 \sigma}(i) a_{i \sigma}^{\dagger} a_{i \sigma}, \quad H_{\mathrm{t}}=H_{L \mathrm{t}}+H_{R \mathrm{t}} .
\end{aligned}
$$

$H_{\mathrm{t}}$ consists of two interaction Hamiltonians, $H_{L \mathrm{t}}$ and $H_{R \mathrm{t}}$ in the following:

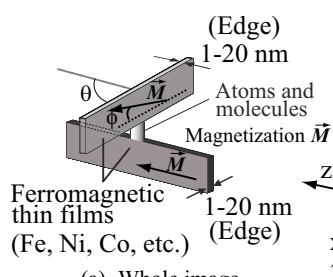

(a) Whole image

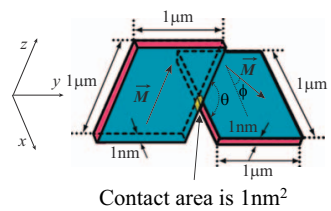

(c) SQCS model with 2D electrodes

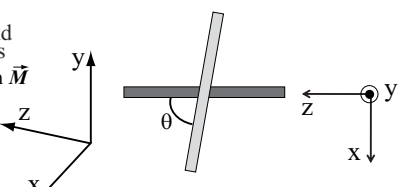

(b) Top view
FIG. 1. (Color) (a) Whole image and (b) top view of SQCS devices and the definition of polar angle $\theta$, and azimuthal angle $\phi$. Schematic illustrations of (c) SQCS device models and (d) the energy diagram for SQCS devices. 


$$
H_{L \mathrm{t}}=\sum_{\boldsymbol{k}, i}\left(V_{L}^{\uparrow \uparrow} c_{L \boldsymbol{k} \uparrow}^{\dagger} a_{i \uparrow}+V_{L}^{\downarrow \downarrow} c_{L \boldsymbol{k} \downarrow}^{\dagger} a_{i \downarrow}+\text { H.c. }\right)
$$

$$
\begin{aligned}
H_{R t}= & \sum_{\boldsymbol{k}, i}\left[\left(V_{R}^{\uparrow \uparrow} e^{i(\phi / 2)} \cos \frac{\theta}{2} c_{R \boldsymbol{k} \uparrow}^{\dagger}-V_{R}^{\uparrow \downarrow} e^{i(\phi / 2)} \sin \frac{\theta}{2} c_{R \boldsymbol{k} \downarrow}^{\dagger}\right) a_{i \uparrow}\right. \\
& +\left(V_{R}^{\downarrow \uparrow} e^{-i(\phi / 2)} \sin \frac{\theta}{2} c_{R \boldsymbol{k} \uparrow}^{\dagger}+V_{R}^{\downarrow \downarrow} e^{-i(\phi / 2)} \cos \frac{\theta}{2} c_{R \boldsymbol{k} \downarrow}^{\dagger}\right) a_{i \downarrow} \\
& + \text { H.c. }] .
\end{aligned}
$$

$H_{\text {Electrodes }}$ is the Hamiltonian of both Ni electrodes, $\epsilon_{\alpha k \uparrow(\downarrow)}$ $=\hbar^{2} \boldsymbol{k}^{2} / 2 m \pm \Delta_{e x}, m$ is the free electron mass, and $\hbar$ is Planck's constant divided by $2 \pi$. The band of itinerant $d$-like electrons of $\mathrm{Ni}$ is assumed to be parabolic with the free electron mass. ${ }^{5}$ The wave vector $\boldsymbol{k}$ is a $2 \mathrm{D}$ vector in $2 \mathrm{D}$ electrodes. The value of $260 \mathrm{meV}$ was used for the exchange splitting $2 \Delta_{e x}$ of $\mathrm{Ni}^{6}{ }^{6} c_{\alpha k \uparrow(\downarrow)}^{\dagger}$ and $c_{\alpha k \uparrow(\downarrow)}$ are creation and annihilation operators for electrons of wave vector $\boldsymbol{k}$ and spin index $\uparrow(\downarrow)$ in $\alpha$ electrode. $\alpha$ indicates the left or right electrode. The creation and annihilation operators obey the standard fermion anticommunication rules. $H_{\text {mole }}$ is the Hamiltonian of a molecule sandwiched between both electrodes, and $\epsilon_{0 \sigma}(i)$ represents the $i$ th energy level of eigenstates of an electron of spin index $\sigma=\uparrow(\downarrow)$ in the molecule as shown in Fig. 1(d). The two energy levels are $\epsilon_{0 \sigma}(1)=0.5 \mathrm{eV}, \epsilon_{0 \sigma}(2)$ $=1 \mathrm{eV}$, estimated from Fermi levels $E_{\mathrm{FL}}, E_{\mathrm{FR}}$ of each electrode, respectively. The Fermi levels $E_{\mathrm{FL}}, E_{\mathrm{FR}}$ of each electrode were assumed to be equal and we used the value of $9.071 \mathrm{eV}$ for the Ni Fermi level. ${ }^{7} a_{i \uparrow(\downarrow)}^{\dagger}$ and $a_{i \uparrow(\downarrow)}$ are creation and annihilation operators for electrons of spin index $\uparrow(\downarrow)$ in the $i$ th energy level. These operators also obey the standard fermion anticommunication rules. Here, the magnetization of the left electrode is pointing to the $\mathrm{z}$ direction, and the magnetization of the right electrode has arbitrary directions represented by polar angle $\theta$, and azimuthal angle $\phi . H_{R(L) t}$ is the transfer Hamiltonian between the sandwiched molecule and the right (left) electrode, $V_{\alpha}^{\sigma \sigma}$ is the spin-dependent electron transfer matrix between an electron of spin index $\sigma$ in the molecule and an electron of spin index $\sigma$ in $\alpha$ electrode. The index of $\sigma(\sigma)$ takes the value of $\uparrow(\downarrow)$. This value $V_{\alpha}^{\sigma \hat{\sigma}}$ determines the coupling strength between each electrode and the molecule. For the sake of simplicity, we assumed that the spin flip scattering does not happen at the interface between the molecule and each electrode. However, in actual devices, the scattering happens by defects or surface roughness at the interface between the molecule and each electrode. If necessary, the effects induced by the scattering can be included in the transfer matrices and densities of states of electrodes, phenomenologically. Notice that this assumption is independent of spin flip processes in the molecule. The spin dependent $I-V$ characteristics from right to left electrode has been studied, taking into consideration both polar angle $\theta$, and azimuthal angle $\phi$. The spin-dependent electron transfer matrices are dependent on each other. The following equations hold after simple consideration:

$$
V_{R}^{\uparrow \uparrow}=V_{R}^{\downarrow \uparrow}, \quad V_{R}^{\downarrow \downarrow}=V_{R}^{\uparrow \downarrow} .
$$

Here, we define the following retarded Green's function of the sandwiched molecule,

$$
G_{i}^{r \sigma \dot{\sigma}}(\epsilon)=-i \int d t \theta(t)\left\langle\left\{a_{i \sigma}(t), a_{i \dot{\sigma}}^{\dagger}(0)\right\}\right\rangle e^{i(\epsilon+i \delta) t},
$$

where $\{\cdots\}$ denotes an anticommutator, $\langle\cdots\rangle$ means the thermodynamic average, $\theta(t)$ is a step function of time $t$, and $\delta$ is an infinitesimal positive quantity. These Green's functions with spin index $\sigma \sigma$ are regarded as matrix elements of $G^{r}(\epsilon)$ which is the $2 \times 2$ matrix in spin space for the retarded Green's function in the sandwiched molecule. $\boldsymbol{G}^{r}(\boldsymbol{\epsilon})$ satisfies the Dyson equation in matrix form as follows:

$$
\boldsymbol{G}^{r}(\epsilon)=\boldsymbol{G}_{0}^{r}(\epsilon)+\boldsymbol{G}_{0}^{r}(\epsilon) \boldsymbol{\Sigma}^{r}(\epsilon) \boldsymbol{G}^{r}(\epsilon),
$$

where the free Green's function $\boldsymbol{G}_{0}^{r}(\boldsymbol{\epsilon})$ and the self-energy $\boldsymbol{\Sigma}^{r}(\boldsymbol{\epsilon})=\boldsymbol{\Sigma}_{R}^{r}(\boldsymbol{\epsilon})+\boldsymbol{\Sigma}_{L}^{r}(\boldsymbol{\epsilon})$ are also $2 \times 2$ matrices in spin space. $\Sigma_{R(L)}^{r}(\epsilon)$ describes the interaction between the molecule and the right (left) electrode. Using Keldysh formalism, ${ }^{8}$ the current $I$ from right to left electrode is described as follows:

$$
\begin{aligned}
& I=\frac{e}{h} \int d \boldsymbol{T} \operatorname{Tr}\left[\boldsymbol{\Gamma}_{L} \boldsymbol{G}^{r} \boldsymbol{\Gamma}_{R} \boldsymbol{G}^{r^{\dagger}}\right]\left(f_{L}-f_{R}\right), \\
& \boldsymbol{\Gamma}_{R}=i\left(\boldsymbol{\Sigma}_{R}^{r}-\boldsymbol{\Sigma}_{R}^{r \dagger}\right), \quad \boldsymbol{\Gamma}_{L}=i\left(\boldsymbol{\Sigma}_{L}^{r}-\boldsymbol{\Sigma}_{L}^{r \dagger}\right),
\end{aligned}
$$

where $f_{R(L)}$ is the Fermi-Dirac distribution function of right (left) electrode, and $\boldsymbol{\Gamma}_{R(L)}$ means the coupling strength matrix between the molecule and right (left) electrode. After tedious manipulations, we can obtain the following matrix elements of the self-energies $\boldsymbol{\Sigma}_{R}^{r}(\boldsymbol{\epsilon})$ and $\boldsymbol{\Sigma}_{L}^{r}(\boldsymbol{\epsilon})$ using the Hamiltonian in Eq. (1):

$$
\begin{aligned}
& \Sigma_{R}^{r \uparrow \uparrow}=\sum_{\boldsymbol{k}}\left[\frac{\left|V_{R}^{\uparrow \uparrow}\right|^{2} \cos ^{2} \frac{\theta}{2}}{\epsilon-\epsilon_{R \boldsymbol{k} \uparrow}+i \delta}+\frac{\left|V_{R}^{\downarrow \downarrow}\right|^{2} \sin ^{2} \frac{\theta}{2}}{\epsilon-\epsilon_{R \boldsymbol{k} \downarrow}+i \delta}\right], \\
& \Sigma_{R}^{r \downarrow}=\sum_{\boldsymbol{k}}\left[\frac{\left|V_{R}^{\downarrow \downarrow}\right|^{2} \cos ^{2} \frac{\theta}{2}}{\epsilon-\epsilon_{R \boldsymbol{k} \downarrow}+i \delta}+\frac{\left|V_{R}^{\uparrow \uparrow}\right|^{2} \sin ^{2} \frac{\theta}{2}}{\epsilon-\epsilon_{R \boldsymbol{k} \uparrow}+i \delta}\right], \\
& \Sigma_{R}^{r \uparrow \downarrow}=\sum_{\boldsymbol{k}} e^{-i \phi}\left[\frac{\left|V_{R}^{\uparrow \uparrow}\right|^{2} \sin \frac{\theta}{2} \cos \frac{\theta}{2}}{\epsilon-\epsilon_{R \boldsymbol{k} \uparrow}+i \delta}-\frac{\left|V_{R}^{\downarrow \downarrow}\right|^{2} \sin \frac{\theta}{2} \cos \frac{\theta}{2}}{\epsilon-\epsilon_{R \boldsymbol{k} \downarrow}+i \delta}\right], \\
& \Sigma_{R}^{r \downarrow \uparrow}=\sum_{\boldsymbol{k}} e^{i \phi}\left[\frac{\left|V_{R}^{\uparrow \uparrow}\right|^{2} \sin \frac{\theta}{2} \cos \frac{\theta}{2}}{\epsilon-\epsilon_{R \boldsymbol{k} \uparrow}+i \delta}-\frac{\left|V_{R}^{\downarrow \downarrow}\right|^{2} \sin \frac{\theta}{2} \cos \frac{\theta}{2}}{\epsilon-\epsilon_{R \boldsymbol{k} \downarrow}+i \delta}\right], \\
& \Sigma_{L}^{r \uparrow}=\sum_{k} \frac{\left|V_{L}^{\uparrow \uparrow}\right|^{2}}{\epsilon-\epsilon_{L k \uparrow}+i \delta}, \quad \Sigma_{L}^{r \downarrow \downarrow}=\sum_{k} \frac{\left|V_{L}^{\downarrow \downarrow}\right|^{2}}{\epsilon-\epsilon_{L k \downarrow}+i \delta} .
\end{aligned}
$$

You can notice that the self-energy $\boldsymbol{\Sigma}_{L}^{r}(\epsilon)$ is a diagonal matrix, and only the off-diagonal elements of the self-energy $\boldsymbol{\Sigma}_{R}^{r}(\boldsymbol{\epsilon})$ have a dependence on azimuthal angle $\phi$. To our knowledge, we have shown azimuthal angle $\phi$ dependence of the self-energy explicitly for the first time. Then, the following matrix elements of the Green's function $\boldsymbol{G}^{r}(\boldsymbol{\epsilon})$ can be obtained using the above self-energy matrix elements and Eq. (8): 


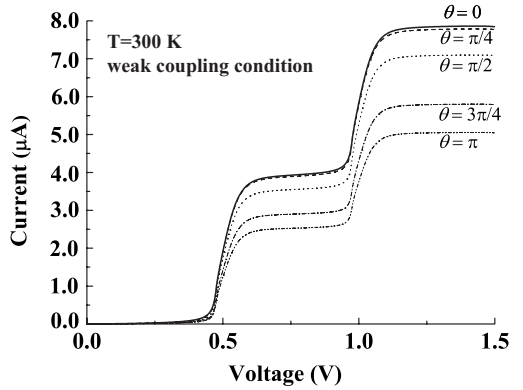

FIG. 2. Calculated results of $I-V$ characteristics for SQCS devices under the weak coupling condition at $300 \mathrm{~K}$.

$$
G_{i}^{r \uparrow \uparrow}=\frac{M_{i}^{\downarrow}}{D_{i}}, G_{i}^{r \uparrow \downarrow}=\frac{\sum^{r \uparrow \downarrow}}{D_{i}}, G_{i}^{r \downarrow \uparrow}=\frac{\sum^{r \downarrow \uparrow}}{D_{i}}, G_{i}^{r \downarrow \downarrow}=\frac{M_{i}^{\uparrow}}{D_{i}},
$$

where

$$
\begin{aligned}
& D_{i}=M_{i}^{\uparrow} M_{i}^{\downarrow}-\Sigma^{r \uparrow \downarrow} \Sigma^{r \downarrow \uparrow}, \\
& M_{i}^{\uparrow}=\epsilon-\epsilon_{0 \uparrow}(i)+i \delta-\Sigma^{r \uparrow \uparrow}, \\
& M_{i}^{\downarrow}=\epsilon-\epsilon_{0 \downarrow}(i)+i \delta-\Sigma^{r \downarrow \downarrow} .
\end{aligned}
$$

Using Eqs. (6) and (9), and the above elements, the current $I$ can be obtained as follows:

$$
\begin{aligned}
I= & \frac{e}{h} \int_{E_{\mathrm{FR}}}^{E_{\mathrm{FR}}+e V} d \epsilon \sum_{i}\left[\Gamma_{L}^{\uparrow \uparrow} \Gamma_{R}^{\uparrow \uparrow}\left(\left|G_{i}^{r \uparrow \uparrow}\right|^{2}+\left|G_{i}^{r \uparrow \downarrow}\right|^{2}\right)\right. \\
& \left.+\Gamma_{L}^{\downarrow \downarrow} \Gamma_{R}^{\downarrow \downarrow}\left(\left|G_{i}^{r \downarrow \downarrow}\right|^{2}+\left|G_{i}^{r \downarrow \uparrow}\right|^{2}\right)\right] \times\left[f\left(\epsilon-e V-E_{\mathrm{FR}}\right)-f(\epsilon\right. \\
& \left.\left.-E_{\mathrm{FR}}\right)\right],
\end{aligned}
$$

where

$$
\begin{aligned}
& \Gamma_{L}^{\uparrow \uparrow}=2 \gamma_{L}^{\uparrow}, \quad \Gamma_{R}^{\uparrow \uparrow}=2\left(\gamma_{R}^{\uparrow \uparrow} \cos ^{2} \frac{\theta}{2}+\gamma_{R}^{\downarrow \downarrow} \sin ^{2} \frac{\theta}{2}\right), \\
& \Gamma_{L}^{\downarrow \downarrow}=2 \gamma_{L}^{\downarrow \downarrow}, \quad \Gamma_{R}^{\downarrow \downarrow}=2\left(\gamma_{R}^{\downarrow \downarrow} \cos ^{2} \frac{\theta}{2}+\gamma_{R}^{\uparrow \uparrow} \sin ^{2} \frac{\theta}{2}\right), \\
& \gamma_{L}^{\uparrow}=\pi D_{L}^{\uparrow}(\epsilon)\left|V_{L}^{\uparrow \uparrow}\right|^{2}, \quad \gamma_{L}^{\downarrow \downarrow}(\epsilon)=\pi D_{L}^{\downarrow}(\epsilon)\left|V_{L}^{\downarrow \downarrow}\right|^{2}, \\
& \gamma_{R}^{\uparrow \uparrow}=\pi D_{R}^{\uparrow}(\epsilon)\left|V_{R}^{\uparrow \uparrow}\right|^{2}, \quad \gamma_{R}^{\downarrow \downarrow}(\epsilon)=\pi D_{R}^{\downarrow}(\epsilon)\left|V_{R}^{\downarrow \downarrow}\right|^{2} .
\end{aligned}
$$

$D_{L}^{\uparrow}(\epsilon), D_{R}^{\uparrow}(\epsilon)\left(D_{L}^{\downarrow}(\epsilon), D_{R}^{\downarrow}(\epsilon)\right)$ are densities of states of the $\uparrow(\downarrow)$ spin electrons in left and right electrodes, respectively. This equation is a general noncollinear spin dependent transport formula with the spin flip process in the molecule, taking into consideration both polar angle $\theta$, and azimuthal angle $\phi$. This formula does not include the azimuthal angle $\phi$ dependence. Although the self-energy $\Sigma^{r \uparrow \downarrow(\downarrow \uparrow)}$ has a dependence on $\phi$, they are canceled out completely. Namely, only the polar angle $\theta$ affects the electronic transport. This fact means that the noncollinear spin transport is determined only by the angle defined by the inner product of two spins. Also, this formula can be widely used for molecular spintronic devices. In most cases the spin flip process does not occur in the molecule. Therefore, $G_{i}^{r \uparrow \downarrow(\downarrow \uparrow)}$ in Eq. (11) vanishes because these Green's functions represent the spin flip process

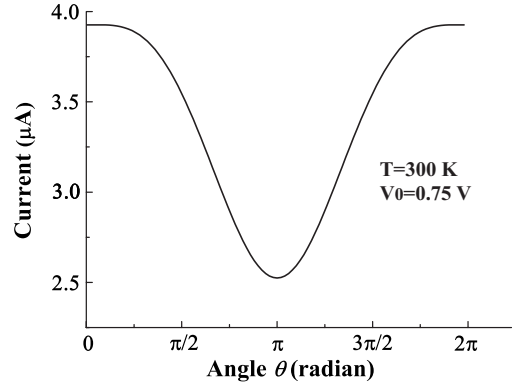

FIG. 3. Angular dependence of the current of SQCS devices at an operating voltage of $0.75 \mathrm{~V}$.

in the molecule. Then, Eq. (11) becomes the following simple formula:

$$
\begin{aligned}
I= & \frac{e}{h} \int_{E_{\mathrm{FR}}}^{E_{\mathrm{FR}}+e V} d \epsilon \sum_{i}\left[\frac{4 \gamma_{L}^{\uparrow}\left(\gamma_{R}^{\uparrow \uparrow} \cos ^{2} \frac{\theta}{2}+\gamma_{R}^{\downarrow \downarrow} \sin ^{2} \frac{\theta}{2}\right)}{\left(\epsilon-\epsilon_{0}(i)\right)^{2}+\gamma_{\uparrow \uparrow}^{2}}\right. \\
& \left.+\frac{4 \gamma_{L}^{\downarrow \downarrow}\left(\gamma_{R}^{\downarrow \downarrow} \cos ^{2} \frac{\theta}{2}+\gamma_{R}^{\uparrow} \sin ^{2} \frac{\theta}{2}\right)}{\left(\epsilon-\epsilon_{0}(i)\right)^{2}+\gamma_{\downarrow \downarrow}^{2}}\right] \times\left[f\left(\epsilon-e V-E_{\mathrm{FR}}\right)\right. \\
& \left.-f\left(\epsilon-E_{\mathrm{FR}}\right)\right],
\end{aligned}
$$

where $\gamma_{\uparrow \uparrow(\downarrow \downarrow)}=\gamma_{L}^{\uparrow \uparrow(\downarrow \downarrow)}+\gamma_{R}^{\uparrow \uparrow(\downarrow \downarrow)} \cos ^{2} \frac{\theta}{2}+\gamma_{R}^{\downarrow \downarrow(\uparrow \uparrow)} \sin ^{2} \frac{\theta}{2}$. The equations $\gamma_{R}^{\uparrow}=\gamma_{L}^{\uparrow \uparrow}$ and $\gamma_{R}^{\downarrow \downarrow}=\gamma_{L}^{\downarrow \downarrow}$ hold from the symmetry. Figure 2 shows calculated results of $I-V$ characteristics for SQCS devices using Eq. (12) under the weak coupling condition. We regard the coupling as weak coupling when the energy of the coupling strength is smaller than that of the ambient temperature $26 \mathrm{meV}$. In this situation, the value of $3.67 \mathrm{meV}$ $(0.92 \mathrm{meV})$ was used for the coupling strength $\gamma_{R}^{\uparrow}\left(\gamma_{R}^{\downarrow \downarrow}\right)$, respectively, assuming that the upspin is the majority spin in both electrodes. Changing polar angle $\theta$, the current gradually decreases because the two Ni magnetic electrodes begin to have components of antiparallel magnetic moments. Figure 3 shows the angular dependence of the current of SQCS devices at an operating voltage of $0.75 \mathrm{~V}$. These results imply that SQCS devices can serve as multivalued memory devices by varying polar angle $\theta$ using microelectromechanical system, etc.

\section{ACKNOWLEDGMENTS}

This research has been partially supported by Special Education and Research Expenses from Post-Silicon Materials and Devices Research Alliance, and also by a Grant-inAid for Scientific Research from Japan Society for the Promotion of Science.

${ }^{1}$ K. Kondo and A. Ishibashi, Jpn. J. Appl. Phys., Part 1 45, 9137 (2006).

${ }^{2}$ H. Kaiju, K. Kondo, and A. Ishibashi, Mater. Res. Soc. Sym. Proc. 961, O05-05 (2007), http://www.mrs.org/s_mrs/bin.asp?CID=7652\&DID $=190653 \& D O C=$ FILE.PDF.

${ }^{3}$ K. Kondo, H. Kaiju, and A. Ishibashi, Mater. Res. Soc. Sym. Proc. 1067, B03-01 (2008), http://www.mrs.org/s_mrs/bin.asp?CID=12455\&DID $=213822 \&$ DOC $=$ FILE.PDF.

${ }^{4}$ K. Kondo, H. Kaiju, and A. Ishibashi, J. Appl. Phys. 105, 07 D522 (2009).

${ }^{5}$ M. B. Stearns, J. Magn. Magn. Mater. 5, 167 (1977).

${ }^{6}$ W. Eberhardt and E. W. Plummer, Phys. Rev. B 21, 3245 (1980).

${ }^{7}$ C. S. Wang and J. Callaway, Phys. Rev. B 15, 298 (1977).

${ }^{8}$ J. Rammer and H. Smith, Rev. Mod. Phys. 58, 323 (1986). 